\title{
In-situ Synthesis of Coral-Like Molybdenum Phosphide (MoP) Microspheres for Lithium-Ion Battery
}

\author{
Xinlong Liu ${ }^{1} \cdot$ Wei Yang ${ }^{1} \cdot$ Zhiting Liu $^{1} \cdot$ Haosen Fan ${ }^{1} \cdot$ Wenzhi Zheng ${ }^{1}$
}

Received: 20 July 2020 / Revised: 19 September 2020 / Accepted: 4 October 2020 / Published online: 2 November 2020

(C) The Chinese Society for Metals (CSM) and Springer-Verlag GmbH Germany, part of Springer Nature 2020

\begin{abstract}
Molybdenum phosphide (MoP) has attracted extensive attention as promising anode candidates for lithium-ion batteries owing to its high specific capacity, low potential range and low polarization. However, severe volume changes and intrinsic low conductivity are major challenges for further application of MoP electrode materials. In this work, a coral-like MoP microsphere encapsulated by N-doped carbon (MoP@NDC) was successfully prepared through annealing the precursor derived from self-polymerization of dopamine with phosphomolybdic acid. The introduction of carbon framework not only serves as matrix to confine MoP nanocrystals from aggregations, but also improves the electrochemical conductivity and facilitates lithium ion or electron transport on the surface of MoP. Such hierarchical structure delivered high discharge capacity of $495 \mathrm{mAh} \mathrm{g}^{-1}$ after 300 cycles with $90.1 \%$ capacity retention, which could be attributed to the synergistic effects of MoP nanoparticles and conductive carbon network. This design strategy shows MoP@NDC electrode with applicable application as anode in lithium-ion battery.
\end{abstract}

Keywords Pseudocapacitance $\cdot$ Coral-like $\cdot$ Lithium-ion batteries (LIBs) $\cdot$ MoP@NDC · Anode

\section{Introduction}

To date, the ever-growing energy demands have urged us to develop advanced energy storage devices, such as sodium-ion battery [1, 2], lithium-sulfur battery [3] and water splitting [4]. However, lithium-ion batteries (LIBs) are considered as one of the attractive rechargeable batteries for practical application [5]. Thus, in order to further optimize LIBs technology, it is imperative to seek for suitable anode materials with satisfying electrochemical performances of ultra-long cycling, high safety and high energy density [6-9]. Compared with conventional graphite anode, transitional metal compounds electrode materials such as metal oxides [10, 11], sulfides $[12,13]$, alloying-type metals [14, 15] and phosphides [16-20] have been regarded as hopeful alternative owing to their high

Available online at http://link.springer.com/journal/40195

Haosen Fan

hsfan@gzhu.edu.cn

Wenzhi Zheng

wenzhizheng@gzhu.edu.cn

1 School of Chemistry and Chemical Engineering, Guangzhou University, Guangzhou 510006, China capacity, natural abundances and large volumetric/gravimetric capacities. Among them, transitional metal phosphides (TMPs) have attracted intensive attention because of its lower operation potentials range $(\sim 1.0 \mathrm{~V})$ than that of oxides/sulfides $(\geq 1.5 \mathrm{~V})$ and low polarization [21-23]. Recently, molybdenum phosphide (MoP) was investigated quite massively in hydrogen evolution reaction because of its high catalytic activity, and superior electrochemical properties [24-26]. Even so, few researches have focused on LIBs with respect to anode material because of its relatively complicated synthesis process. In fact, MoP can also serve as promising anode with relatively high-theoretical specific capacity of $632 \mathrm{mAh}$ $\mathrm{g}^{-1}$. Nevertheless, MoP also suffers from severe irreversible volume changes during extensive lithiation/delithiation process. This inevitable obstacle results in structural pulverization of the electrode, which causes inferior cycling stability and rate capability. To address those issues, researchers have proposed several strategies, which is based on enabling faster ions diffusion kinetics and improving structural stability. Typically, downsizing MoP particles into nanoscale is an effective approach to mitigate structural strains caused by $\mathrm{Li}^{+}$ insertion/extraction and shorten $\mathrm{Li}^{+}$diffusion path by improving electrode-electrolyte contact areas [27, 28]. In addition, building a highly conductive composite by incorporating 
carbonaceous materials is another practical method to boost MoP-based anode electrochemical performances. The conductive matrix not only elevates faster electron movement around active materials, but also it serves as a volume buffer to relieve structural strains, which is conducive to battery performances [17, 29-32]. However, coupling carbonaceous materials and nanoparticles together could be difficult in terms of preparation. Moreover, when applied to LIBs as anode materials, these hybrids so far have not yet achieved ideal electrochemical performances [33, 34].

Polyoxometalates (POMs), a kind of polymetallic oxycluster compounds are typically consisted of three-dimensional anionic metal oxide clusters in their high valence state [35-37]. The versatility of POMs allows for various applications including polymer dopants [38], catalysis [39], ion battery components [40] and geochemistry [41] due to their controllable morphologies. The most commonly used POMs are Keggin structure $\left(\left[X M_{12} \mathrm{O}_{40}\right]^{n-}\right)$ and Wells-Dawson structure $\left(\left[X_{2} M_{18} \mathrm{O}_{62}\right]^{n-}\right.$ ), where $X$ is typically $\mathrm{Si}$ or $\mathrm{P}$ and $M$ is typically a group V metal (such as Mo or W). Dopamine (DA), on the other hand, a type of neurotransmitter from mammalian central nervous system, can be coated on any surface of substrate including organic and inorganic materials through self-oxidation [42]. It has been reported that it can self-assemble with polyoxoanions to form organic-inorganic hybrids [43, 44]. Yet, there only have been few attempts to fabricate 3D hierarchical multifunctional structure based on such organic-inorganic precursor used in energy storage field.

In this work, a coral-like 3D microsphere with MoP nanoparticles confined in conductive $\mathrm{N}$-doped carbon matrix was successfully synthesized through two steps of annealing under subsequent heat treatment in $\mathrm{N}_{2}$ and $\mathrm{H}_{2} / \mathrm{Ar}$, respectively. Mobased precursor was formed through self-polymerization of dopamine with phosphomolybdic acid in Tris solution. In such approach, it can confine MoP nanocrystals inside N-doped carbon-layer decomposed from dopamine after annealing. This special hierarchical structure conspicuously reduces MoP to nanocrystals from agglomeration during charge/discharge process, which can significantly shorten $\mathrm{Li}^{+}$diffusion path and increase surface area. Furthermore, the incorporated conductive carbon greatly offsets the shortage of poor electron transport in MoP crystals. Benefiting from 3D hierarchical structure and carbon coating, the MoP@NDC electrode obtained outstanding electrochemical performance, suggesting its practicable application for LIBs.

\section{Experimental}

\subsection{Materials Synthesis}

Preparation of the Mo-hydrids: Molybdenum-based polydopamine-heteropolyacid hybrids (denoted as Mo-hybrids) microspheres was prepared according to the following procedure: Firstly, $100 \mathrm{mg}$ dopamine hydrochloride was added into Tri-buffer solution $(\mathrm{PH}=8.5)$ and stirred for $20 \mathrm{~min}$ to form a clear suspension. After that, $100 \mathrm{mg}$ phosphomolybdic acid (denoted as $\mathrm{PMo}_{12}$ ) was added and the solution was kept stirring for $24 \mathrm{~h}$ at room temperature. After being centrifugated and washed with pure water and ethanal several times, the dark brown precipitates were collected and dried overnight at $60^{\circ} \mathrm{C}$.

Preparation of the MoP@NDC: The above as-prepared Mo-hybrids precursor was annealed in nitrogen atmosphere at $600{ }^{\circ} \mathrm{C}$ for $2 \mathrm{~h}$ to obtain $\mathrm{MoO}_{2} @ \mathrm{NDC}$. After that, $100 \mathrm{mg}$ immediate product and $1.0 \mathrm{~g} \mathrm{NaH}_{2} \mathrm{PO}_{4}$ in two different corundum crucibles were placed in a quartz tube with $\mathrm{NaH}_{2} \mathrm{PO}_{4}$ at the upper stream in the furnace. The final products were prepared by heating to $800{ }^{\circ} \mathrm{C}$ under $5 \% \mathrm{H}_{2} /$ Ar atmosphere for $2 \mathrm{~h}$ with a heating rate of $5{ }^{\circ} \mathrm{C} / \mathrm{min}$. For comparison, the bulk MoP was also prepared in the similar annealing process without addition of dopamine.

\subsection{Characterizations}

Crystal structure of the synthesized materials was characterized by X-ray diffraction patterns (XRD, D8Rigaku9000, $\mathrm{Cu} K \alpha$ radiation) in a $2 \theta$ range from $5^{\circ}$ to $80^{\circ}$. The microstructural morphology of prepared sample was analyzed by field emission scanning electron microscopy (FESEM, JEOL JSE-7001F). The element valence state and existence were studied by X-ray photoelectron spectroscopy (XPS, AXIS Ultra DLD, AlKo radiation).

\subsection{Electrochemical Measurements}

Electrochemical properties were tested on CR-2032 coin half-cells assembled in argon-filled glove box with moisture and oxygen contents below $0.1 \mathrm{ppm}$. The working electrodes were prepared by mixing active materials, the conductive agent (super P) and binder (sodium alginate) in a weight ratio of $7: 2: 1$. The resulting slurry was directly coated onto copper foil with mass loading of about $1.0 \mathrm{mg} /$ $\mathrm{cm}^{2}$ and dried overnight in a vacuum oven at $60{ }^{\circ} \mathrm{C}$. Pure lithium sheet was used as counter electrode and polypropylene membrane as separator. Electrolyte was $1 \mathrm{M} \mathrm{LiPF}_{6}$ dissolved in mixed solution of ethylene carbonate/diethyl carbonate (EC/DEC; $1: 1 \mathrm{v} / \mathrm{v})$. The galvanostatic discharge/ charge tests were carried out on NEWARE battery tester at voltage window ranging from 0.01 to $3.0 \mathrm{~V}$ at $25^{\circ} \mathrm{C}$. Cyclic voltammetry (CV) was measured on IviumnStat multifunctional working station with potential range from 0.01 to $3.0 \mathrm{~V}$. 


\section{Results and Discussion}

The facile synthesis procedure of coral-like 3D MoP@ NDC microspheres is schematically illustrated in Fig. 1. In short, Mo-hybrids were synthesized as a precursor and followed by two steps of annealing procedure. Firstly, the Mo-polydopamine composites were fabricated through a self-polymerization with $\mathrm{PMo}_{12}$ to generate a coral-like microsphere. Then, above product was reduced in nitrogen atmosphere to yield $\mathrm{MoO}_{2} @$ NDC. During thermal reduction procedure, $\mathrm{MoO}_{2}$ could easily tend to aggregate at high calcination temperature in order to lower the high surface energy. However, with the advantage of adding carbonaceous materials, the $\mathrm{N}$-doped carbon pyrolyzed from polydopamine can serve as supporting substrates to prevent losing surface area. The final product MoP@ NDC microsphere was prepared through a phosphorization process via anions exchange by substituting oxygen to phosphorus [45]. The amorphous carbon played an important role in keeping the MoP nanoparticles from further aggregations, thus reserving the structural integrity of $3 \mathrm{D}$ microsphere architecture at high temperature.

The crystal structure and planes of MoP@NDC have been confirmed by XRD. As shown in Fig. 2a, the XRD pattern of MoP@NDC shows serval diffraction peaks at $28.2^{\circ}, 32.1^{\circ}, 43.0^{\circ}, 56.9^{\circ}$ and $64.9^{\circ}$, corresponding to (001), (100), (101), (002) and (111) lattice planes of MoP crystalline, respectively. All diffraction peaks can be assigned to hexagonal MoP (JCPDS card No: 24-0771, space group $P-6 m 2$ ). In this hexagonal structure (Fig. 2b), the center Mo atom is connected with six $\mathrm{P}$ atoms located at each corner of triangular prism. Furthermore, there are no impurity peaks to be observed, indicating good crystallinity of MoP and successful transformation into MoP phase from Mo-based precursor. Because of the existence of amorphous carbon, graphitic carbon was hardly detected at around $26.5^{\circ}$ indexed to (002) plane. As shown in Fig. 3a, there are two obvious peaks at around $1342 \mathrm{~cm}^{-1}$ (D band, disordered $s p^{2}$ carbon) and $1581 \mathrm{~cm}^{-1}$ (G band, graphitic $s p^{2}$ carbon) with $I_{\mathrm{D}} / I_{\mathrm{G}}$ calculated to be 1.07, demonstrating the both distribution amorphous and graphitic carbon from thermal decomposition of polydopamine.

XPS was performed to study the element valence state and existence on the surface of MoP@NDC. The survey XPS spectrum in Fig. $3 \mathrm{~b}$ presents the existence of Mo, P, $\mathrm{C}, \mathrm{N}$ and $\mathrm{O}$ elements. The two peaks (Fig. 3c) centered at 236.1 and $233.0 \mathrm{eV}$ are ascribed to $\mathrm{Mo}^{6+} 3 d_{3 / 2}$ and $\mathrm{Mo}^{6+}$ $3 d_{5 / 2}$ states, respectively, resulting from the partial oxidation on the surface of MoP when exposed in air [46]. The other peaks occurred at 232.3 and $229.2 \mathrm{eV}$ are attributed to $\mathrm{Mo}^{4+}$

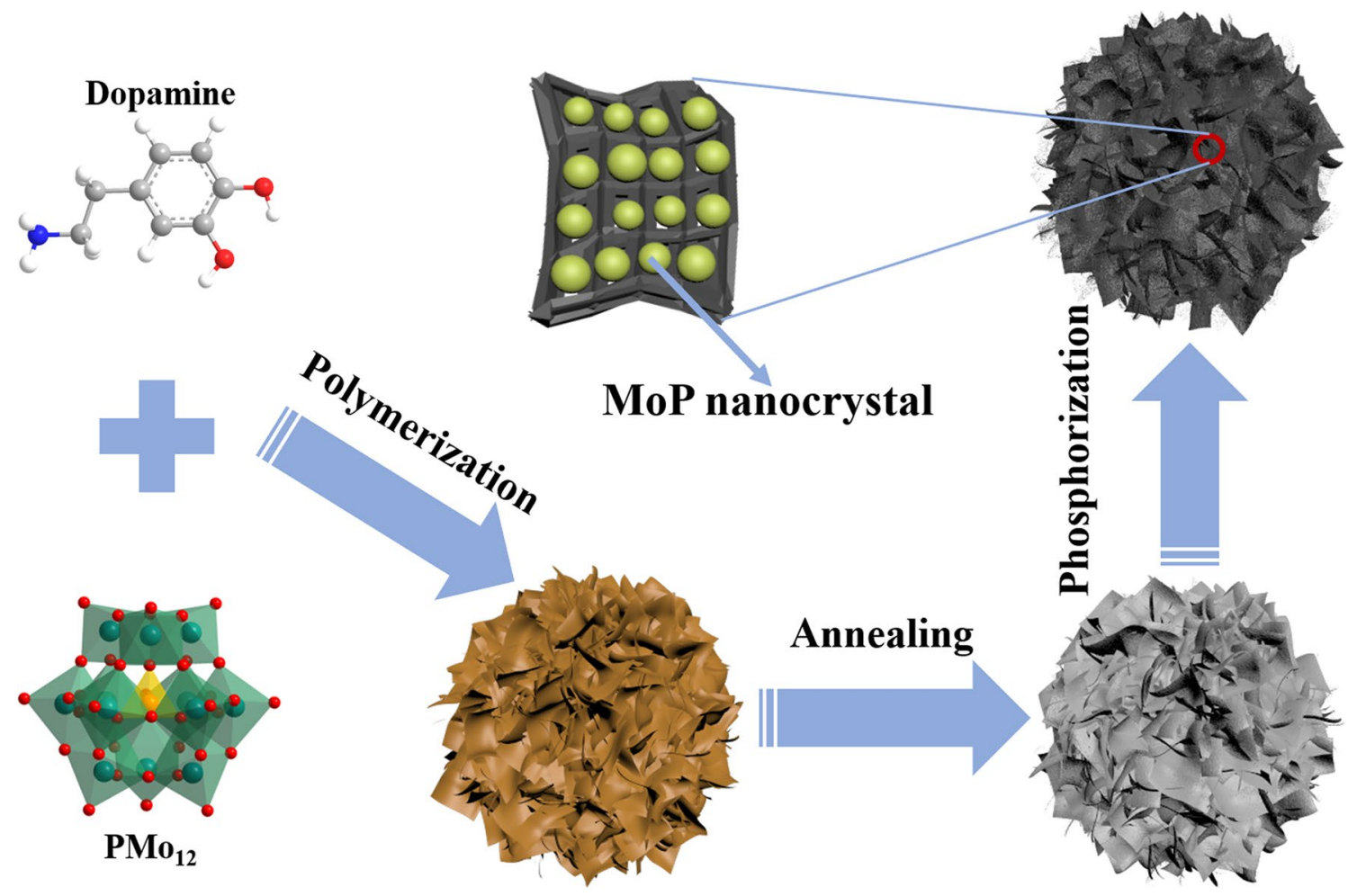

Fig. 1 Schematic illustration of the preparation of MoP@NDC 

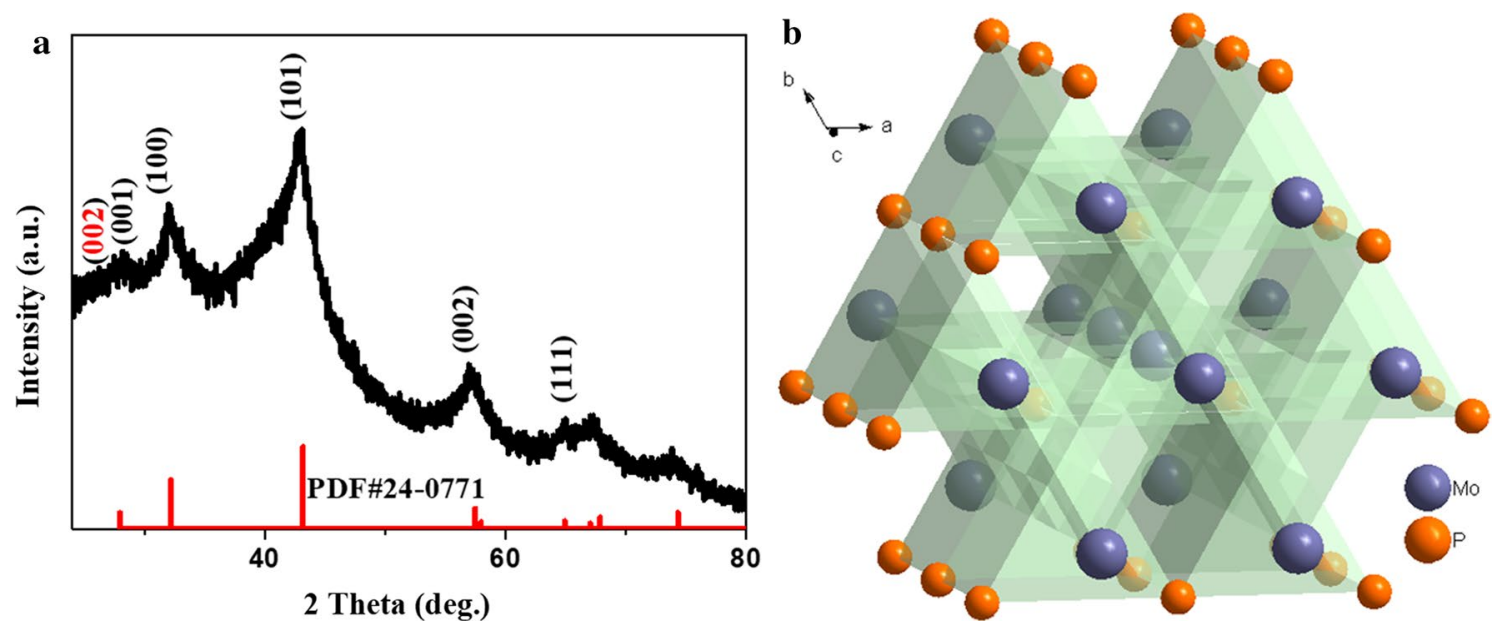

Fig. 2 a XRD pattern of as-prepared MoP@ NDC; $\mathbf{b}$ hexagonal structure of MoP

$3 d_{3 / 2}$ and $\mathrm{Mo}^{4+} 3 d_{5 / 2}$ due to incomplete phosphidation of the slight $\mathrm{MoO}_{2}$ content. Additionally, the fitting peaks (Fig. 3d) at $133.5 \mathrm{eV}$ and $134.7 \mathrm{eV}$, respectively, are assigned to $\mathrm{P}$ $2 p_{3 / 2}$ and $\mathrm{P} 2 p_{1 / 2}$ of $\mathrm{P}-\mathrm{Mo}$ bond [47]. The peaks at $137.9 \mathrm{eV}$ belong to the $\mathrm{P}-\mathrm{O}$ bond of the $\mathrm{PO}_{3}{ }^{3-}$ species, which was caused by minor oxidation of phosphorus remaining on the surface of MoP microsphere. There are three components fitted in spectrum C $1 s$ (Fig. 3e). The major peak located in $284.1 \mathrm{eV}$ corresponds to graphite carbon atoms. Meanwhile, the other two peaks at $285.1 \mathrm{eV}$ and $286.8 \mathrm{eV}$ are explained to be $\mathrm{C}-\mathrm{O}$ and $\mathrm{O}=\mathrm{C}-\mathrm{O}$ bonds, respectively [48]. The $\mathrm{N}$ $1 s$ spectra in Fig. $3 \mathrm{f}$ are identified with three peaks with binding energy of $396.3 \mathrm{eV}, 398.1 \mathrm{eV}$ and $400.0 \mathrm{eV}$, where they are assigned to pyridinic- $\mathrm{N}$, pyrrolic- $\mathrm{N}$ and graphitic$\mathrm{N}$, respectively [49]. It is noted that the peak centered at $394.7 \mathrm{eV}$ could pertain to the Mo-N coupling bonding [50]. The nitrogen doping was expected to improve the electronic conductivity and provide more active sites for ions diffusion [49], which boosts faster $\mathrm{Li}^{+}$transport kinetic synergistic effects with carbon layer. The XPS results demonstrate further that MoP@NDC was successfully prepared by twosteps annealing process.

The morphology and microstructure of Mo-hybrids and MoP@NDC were analyzed by SEM. As shown in Fig. 4a-b, the Mo-hybrids are uniform spheres with diameter about 2-4 $\mu \mathrm{m}$. From the high magnification image (Fig. 4c), it can be clearly observed that $2 \mathrm{D}$ nanosheets are interconnected together to form a coral-like 3D spherical framework. The MoP@NDC (Fig. 4d-f) after phosphorization process still maintains coral-like microsphere morphology in which MoP nanocrystals are evenly dispersed on the N-doped carbon. This well-distributed structure creates more reactive sites for lithiation reaction between electrode and electrolyte. The formation of carbon nanoparticles helps suppress structural stain and increase electronic conductivity as well, leading to elevating electrochemical properties of MoP@NDC. It is noteworthy that through subsequent heat treatment, the nanosheets (Fig. 4f) folded up inwards mildly because of the high surface energy without obvious aggregations, which is beneficial from carbon substrate.

Cyclic voltammogram (CV) curve was performed to evaluate the electrochemical performance of MoP@NDC electrode. As shown in Fig. 5a, CV curve was conducted at a scan rate of $0.1 \mathrm{mV} \mathrm{s}^{-1}$ in a voltage window of $0.01-3.0 \mathrm{~V}$. A clear reduction peak appeared at around $0.47 \mathrm{~V}$ in the first cathodic sweep, which can be attributed to the formation of solid electrolyte interface (SEI) film on the electrode surface. After subsequent cycles, this reduction peak disappeared, but two distinct pair of redox peaks at 1.23/1.68 and 1.91/2.26 V appeared, which can be ascribed to the lithiation/delithiation process of $\mathrm{Li}_{x} \mathrm{MoP}$ [51]. It is worth mentioning that after first CV sweep, two redox peaks show no major peak shift and almost overlap very well, implying its high reversibility and stability of MoP@NDC electrode during lithiation/delithiation process.

The galvanostatic discharge and charge profiles of 1 st, 2nd, 3rd, 10th and 50th for MoP@NDC microsphere at a current density of $0.2 \mathrm{~A} \mathrm{~g}^{-1}$ are presented in Fig. 5b. MoP@ NDC exhibits a discharge/charge capacity of 1037/677 mAh $\mathrm{g}^{-1}$ in first cycle, respectively, giving an initial columbic efficiency (CE) of $65.3 \%$. The relatively low CE due to large capacity loss in 1st cycle was usually caused by the formation of SEI film and the decomposition of electrolyte. After second to fiftieth cycles, the columbic efficiency of MoP@ NDC electrode increased from 87.4 to $97.4 \%$, demonstrating that a stable SEI layer was formed and the lithium ions extraction/insertion behavior was highly irreversible during cycling process.

The current densities from $0.1 \mathrm{~A} \mathrm{~g}^{-1}$ to $5.0 \mathrm{~A} \mathrm{~g}^{-1}$ were tested to analyze the rate capabilities of MoP@NDC and 

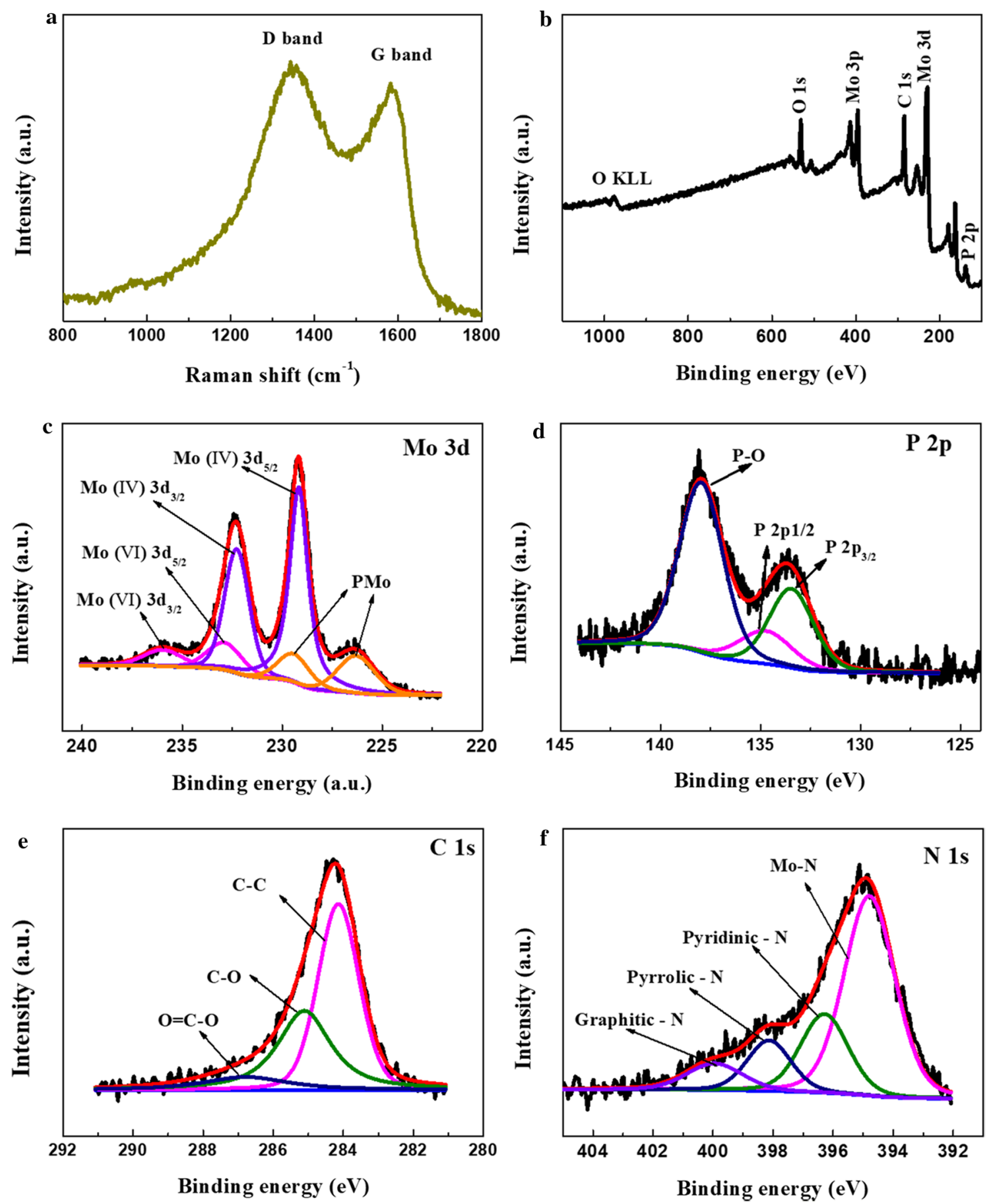

Fig. 3 a Raman spectrum of MoP@NDC; surface composition analysis of MoP/NDC: b XPS full spectrum; high-resolution spectra of $\mathbf{c}$ Mo 3d, d P $2 p$, e C $1 s$ and $\mathbf{f} \mathrm{N} 1 s$

bulk MoP anode. Figure 5c depicts that different reversible specific capacities obtained are 596, 566, 477, 410, 328 and $214 \mathrm{mAh} \mathrm{g}^{-1}$ at the current densities of $0.1,0.2$, $0.5,1.0,2.0$ and $5.0 \mathrm{~A} \mathrm{~g}^{-1}$, respectively. When switched back to small current density of $0.1 \mathrm{~A} \mathrm{~g}^{-1}$, it is remarkable that it still retains high-reversible specific capacity of $587 \mathrm{mAh} \mathrm{g}^{-1}$, signifying prominent rate performance of as-prepared composite. On the contrary, the bulk MoP only achieved $259 \mathrm{mAh} \mathrm{g}^{-1}$ at $0.1 \mathrm{~A} \mathrm{~g}^{-1}$, albeit $602 \mathrm{mAh}$ $\mathrm{g}^{-1}$ in the initial cycle. Galvanostatic discharge/charge curves of MoP@NDC at different current densities were presented in Fig. 5d. There are two obvious redox plateaus centered at around 1.36/1.61 V and 1.85/2.10 V, which are in well consistent with $\mathrm{CV}$ test result. The charge/ 

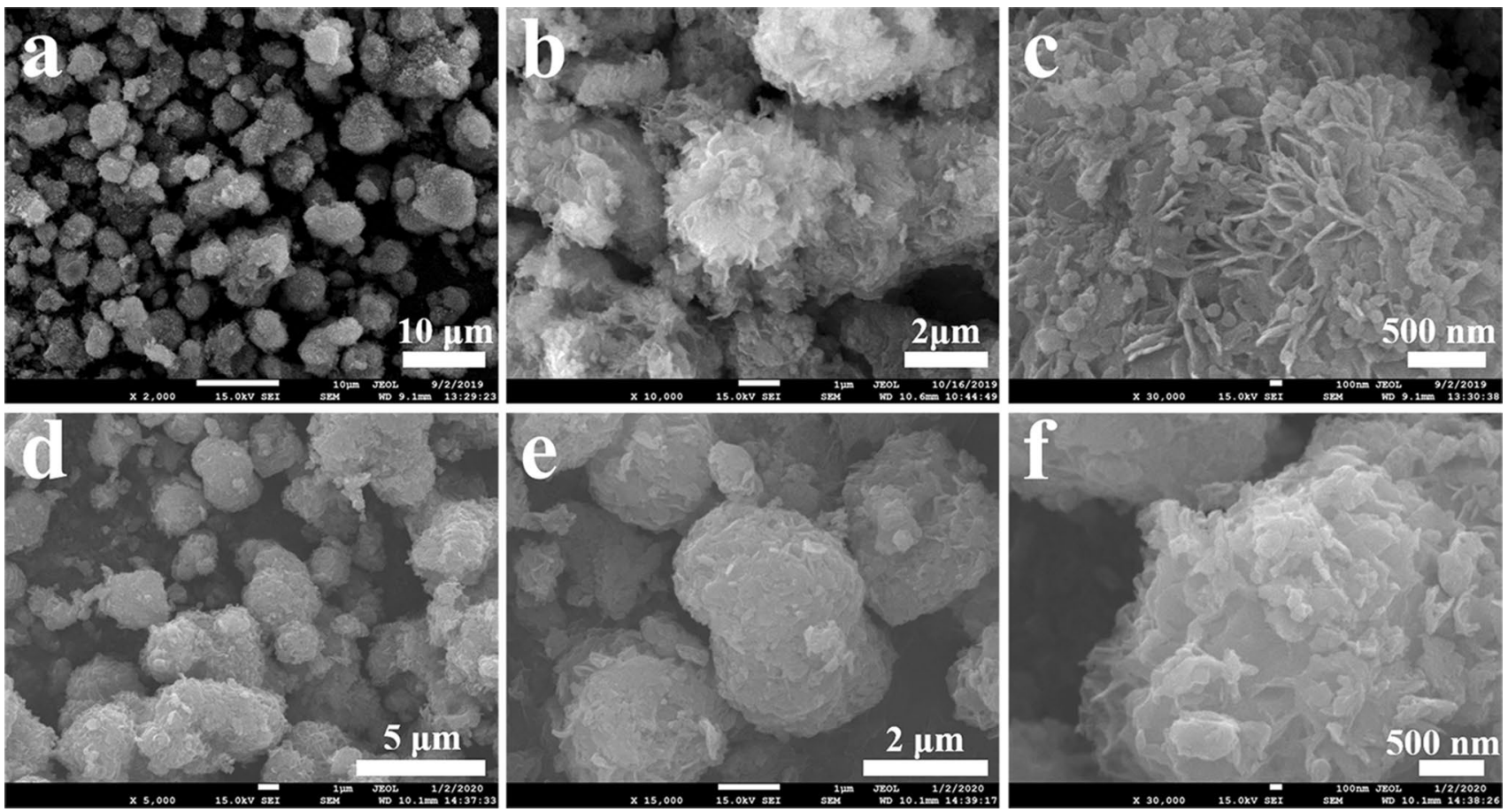

Fig. 4 a-c SEM images of Mo-hybrids; d-f SEM images of MoP@NDC

discharge platforms are still very distinct even at high current density of $2.0 \mathrm{~A} \mathrm{~g} \mathrm{~g}^{-1}$.

Long-term cycling performances MoP@NDC and bulk MoP electrode are displayed in Fig. 5e at a high rate of $1.0 \mathrm{~A}$ $\mathrm{g}^{-1}$. The bulk MoP simply attained relatively low discharge capacity of about $150 \mathrm{mAh} \mathrm{g}^{-1}$ due to the inaccessibility of electrolyte. Comparatively, MoP@NDC electrode demonstrates superior properties with stable cycling capability and high reversible capacity at high current density, which maintained a $90.1 \%$ cycling retention after 300 cycles with $495 \mathrm{mAh} \mathrm{g}^{-1}$ almost as high as its theoretical specific capacity $\left(632 \mathrm{mAh} \mathrm{g}^{-1}\right)$. The $\mathrm{N}$-doped carbon substrate, on the one hand, promotes $\mathrm{Li}^{+}$diffusion to improve ionic transfer kinetics. On the other hand, it serves as carbon matrix and spacer to keep MoP nanoparticles from aggregation and pulverization as well during lithiation/delithiation process, implying its structural stability.

To further understand insight about kinetic reaction mechanism of MoP@NDC, CV profiles (Fig. 6a) were measured under different scan rates from 0.1 to $1.0 \mathrm{mV}$. The shape of CV curves shows slight change while the current density increased in accordance with scan rates, indicating the excellent rate performance. The energy storage mechanism in batteries can be divided into two parts (pseudocapacitance contribution and diffusion-controlled), which are decided by the ratio of each capacity contribution [52]. The current response $(i)$ measured at specific scan rate $(v)$ can be expressed as $i=a v^{b}$ based on power law. Value $b$ varying from 0.5 to 1.0 can be obtained by plotting the slope of $\log (v)-\log (i)$. If the $b$ value is calculated to be close to 0.5 , the electrochemical reaction is dominated by diffusioncontrolled, while if $b$ value is inclined to 1.0 , then capacitive contribution is majority. As shown in Fig. 6b, cathodic peak and anodic peaks have $b$ value of $0.77,0.80,0.87$ and 0.91 , respectively, implying large contribution from pseudocapacitance. The relationship between capacitive contribution $\left(k_{1} v\right)$ and diffusion-controlled $\left(k_{2} v^{1 / 2}\right)$ is given in the equation: $i(\mathrm{~V})=k_{1} v+k_{2} v^{1 / 2}$ or $i(\mathrm{~V}) / v^{1 / 2}=k_{1} v^{1 / 2}+k_{2}$, in which $k_{1}$ value can be easily attained from the $i(\mathrm{~V}) / v^{1 / 2}-v^{1 / 2}$ plot to determine capacitive contribution [53]. As an example, as illustrated in Fig. 6c, the total contribution (pink region) calculated from CV profiles recorded at a scan rate of $1.0 \mathrm{mV} \mathrm{s}^{-1}$ is $72 \%$. Figure $6 \mathrm{~d}$ shows that the capacitive contributions are $53.32 \%, 56.27 \%, 59.22 \%, 67.13 \%$ and $72.00 \%$, respectively, at different scan rates from 0.2 to $1.0 \mathrm{eV}$ based on same calculation method. It is obvious that the pseudo-capacitive behavior does occupy substantial part of total storage capacity of MoP@NDC anode.

\section{Conclusion}

In general, the coral-like MoP@NDC microsphere derived from Mo-based dopamine precursor, was synthesized via a consecutive annealing procedure. When tested as LIBs anode materials, it exhibits excellent electrochemical 

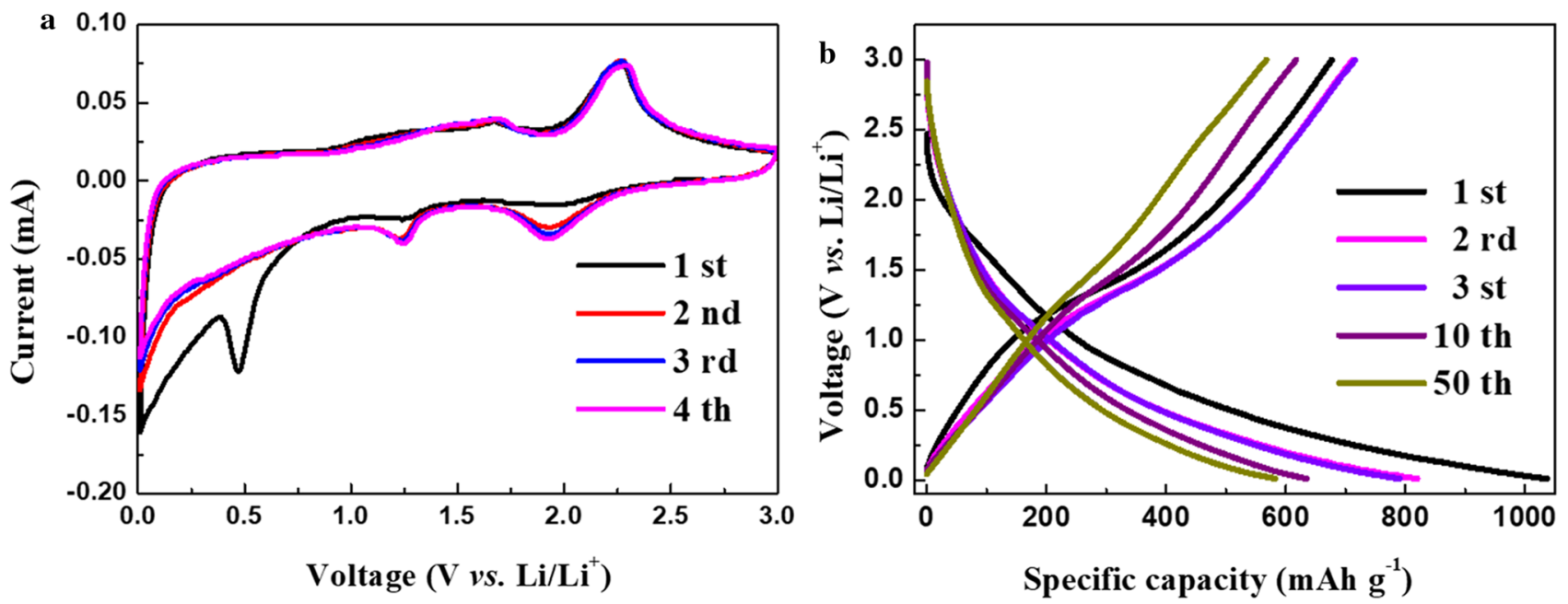

c
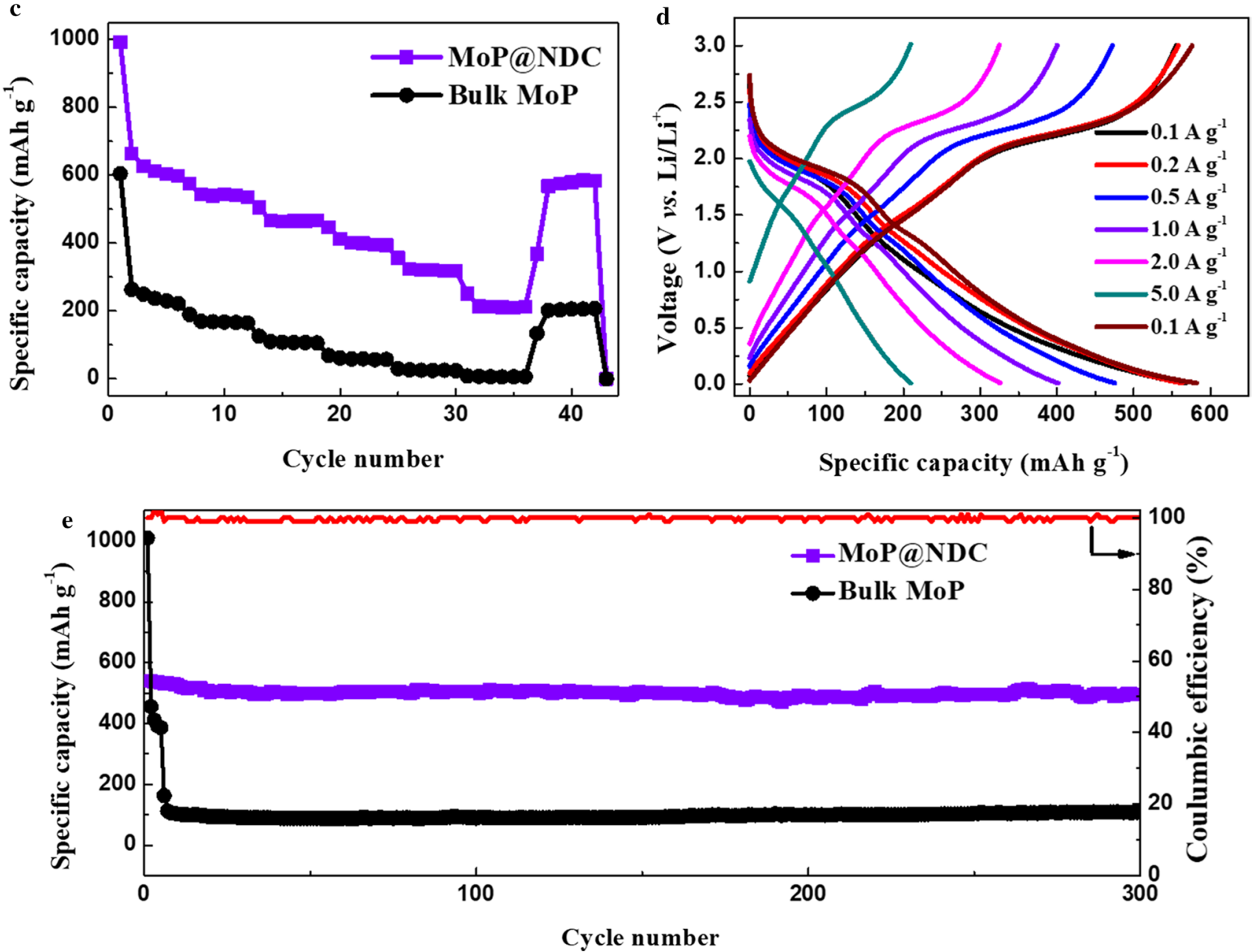

Fig. 5 a CV curves at a scan rate of $0.1 \mathrm{mV} \mathrm{s}^{-1}$ within a potential range of $0.01-3 \mathrm{~V}$; $\mathbf{b}$ galvanostatic charge/discharge profiles; $\mathbf{c}$ rate capability; d charge/discharge curves at various current densities; e cycling performance at $1.0 \mathrm{~A} \mathrm{~g}^{-1}$

properties with both superior cycling and rate capability. In this unique structure, MoP nanocrystallinities were uniformly distributed into $\mathrm{N}$-doped carbon matrix, where it not only promotes conductivity of the materials, but also greatly reduces aggregations of MoP nanocrystals. Furthermore, when coupled with dopamine carbon source, it generated 

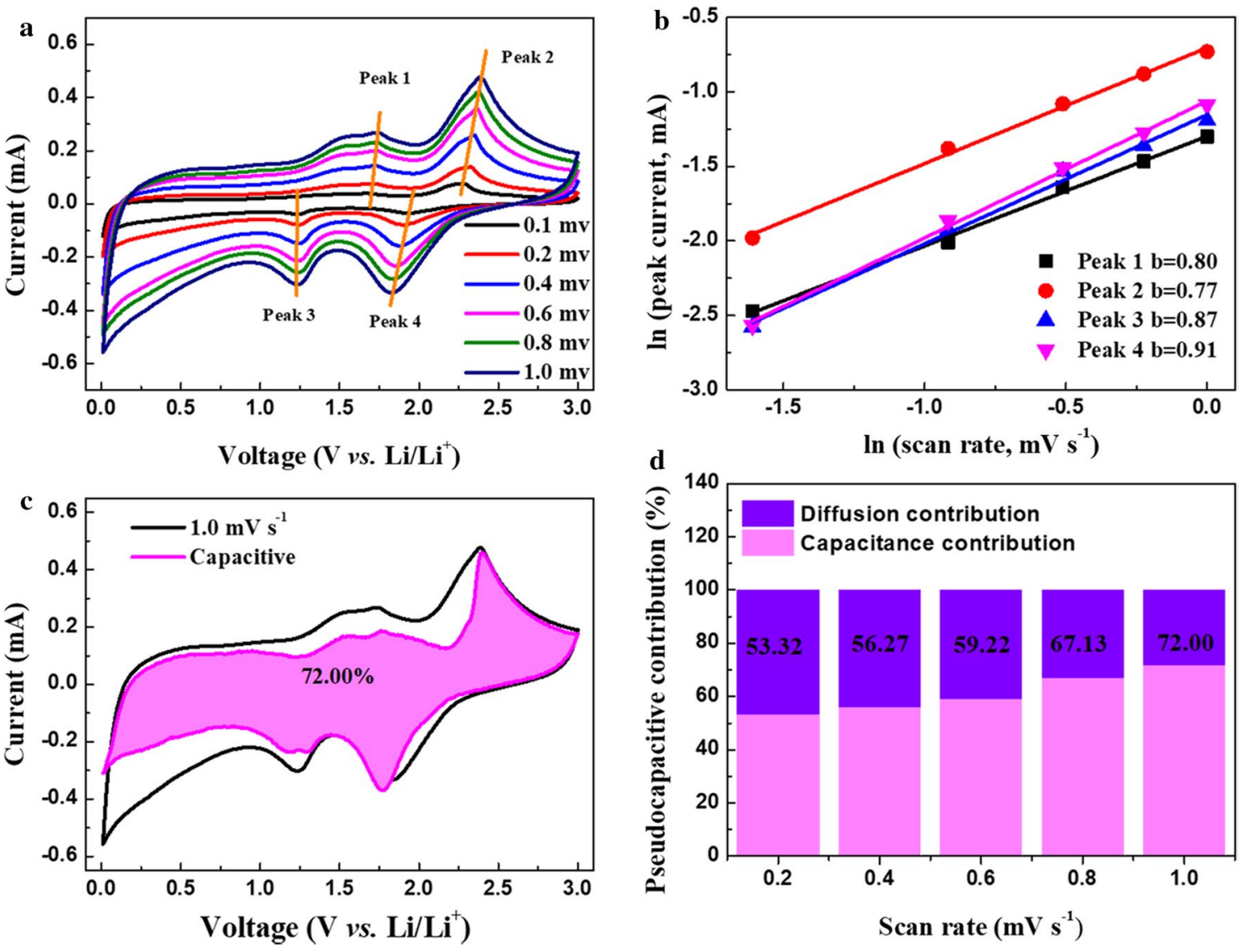

Fig. 6 a CV curves at different sweep rates; $\mathbf{b} b$ values at different redox states; $\mathbf{c}$ contribution of capacitive current (pink region) at the sweep rate of $1.0 \mathrm{mV} \mathrm{s}^{-1}$; $\mathbf{d}$ bar chart pseudocapacitive contribution at different scan rates

a thin carbon layer on MoP after annealing process, thus alleviating large volume change caused by extensive lithium insertion/extraction. Due to these merits, MoP@NDC delivers a reversible capacity of $495 \mathrm{mAh} \mathrm{g}^{-1}$ after 300 cycles and outstanding rate capability. This facile preparing approach demonstrates an effective strategy for fabricating molybdenum phosphide/carbon composites with enhanced electrochemical properties as anode material in LIBs.

Acknowledgements This work was supported by the Natural Science Foundation of Guangdong Province of China (No. 2018A030313516) and the Science and Technology Planning Project of Guangzhou (No. 201804010449).

\section{References}

[1] H. Fan, H. Yu, Y. Zhang, J. Guo, Z. Wang, H. Wang, N. Zhao, Y. Zheng, C. Du, Z. Dai, Q. Yan, J. Xu, Energy Storage Mater. 10, 48 (2018)

[2] Y.Y. Wang, H.S. Fan, B.H, Hou, X.H. Rui, Q.L. Ning, Z. Cui, J.Z. Guo, Y. Yang, X.L. Wu. J. Mater. Chem. A 6, 22966 (2018)

[3] Y. Chen, S. Niu, W. Lv, C. Zhang, Q. Yang, Chin. Chem. Lett. 30, 521 (2019)
[4] H. Fan, H. Yu, Y. Zhang, Y. Zheng, Y. Luo, Z. Dai, B. Li, Y. Zong, Q. Yan, Angew. Chem. Int. Ed. 56, 12566 (2017)

[5] Y. Lin, Z. Qiu, D. Li, S. Ullah, Y. Hai, H. Xin, W. Liao, B. Yang, H. Fan, J. Xu, C. Zhu, Energy Storage Mater. 11, 67 (2018)

[6] R. Sun, Z.X. Qin, Z.Y. Li, H.S. Fan, S.J. Lu, Dalton Trans. 49, $14237(2020)$

[7] X. Yang, Y.Y. Wang, B.H. Hou, H.J. Liang, X.X. Zhao, H. Fan, G. Wang, X.L. Wu, Acta Metall. Sin. -Engl. Lett. (2020). https:// doi.org/10.1007/s40195-020-01001-7

[8] L. Zhou, K. Zhang, Z. Hu, Z. Tao, L. Mai, Y.M. Kang, S.L. Chou, J. Chen, Adv. Energy Mater. 8, 1701415 (2018)

[9] D. Liu, W. Li, Y. Zheng, Z. Cui, X. Yan, D. Liu, J. Wang, Y. Zhang, H. Lu, F. Bai, J. Guo, X. Wu, Adv. Mater. 30, 1706317 (2018)

[10] H.J. Liang, J.Y. Wu, M.Q. Wang, H.S. Fan, Y.F. Zhang, Dalton Trans. 49, 13311 (2020)

[11] X.L. Liu, J.Y. Wu, M.Q. Wang, H.S. Fan, Y.F. Zhang, Ceram. Int. 46, 27608 (2020)

[12] C. Zhu, P. Kopold, W. Li, P.A. van Aken, J. Maier, Y. Yu, Adv. Sci. 2, 1500200 (2015)

[13] F. Chen, L. Wu, Z. Zhou, J. Ju, Z. Zhao, M. Zhong, T. Kuang, Chin. Chem. Lett. 30, 197 (2019)

[14] S. Fang, L. Shen, S. Li, G.T. Kim, D. Bresser, H. Zhang, X. Zhang, J. Maier, S. Passerini, ACS Nano 13, 9511 (2019)

[15] L. Zhang, Y. Ding, J. Song, Chin. Chem. Lett. 29, 1773 (2018)

[16] J. Mao, X. Fan, C. Luo, C. Wang, ACS. Appl. Mater. Interfaces 8, 7147 (2016) 
[17] C. Wu, P. Kopold, P.A. van Aken, J. Maier, Y. Yu, Adv. Mater. 29, 1604015.1 (2017)

[18] J.W. Hall, N. Membreno, J. Wu, H. Celio, R.A. Jones, K.J. Stevenson, J. Am. Chem. Soc. 134, 5532 (2012)

[19] G. Xia, J. Su, M. Li, P. Jiang, Y. Yang, Q. Chen, J. Mater. Chem. A 5, 10321 (2017)

[20] B. Wang, K. Chen, G. Wang, X. Liu, H. Wang, J. Bai, Nanoscale 11, 968 (2019)

[21] Y. Zhao, L.P. Wang, M.T. Sougrati, Z. Feng, Y. Leconte, A. Fisher, M. Srinivasan, Z. Xu, Adv. Energy Mater. 7, 1601424 (2017)

[22] F. Zhao, N. Han, W. Huang, J. Li, H. Ye, F. Chen, Y. Li, J. Mater. Chem. A 3, 21754 (2015)

[23] W. Li, L. Gan, K. Guo, L. Ke, Y. Wei, H. Li, G. Shen, T. Zhai, Nanoscale 8, 8666 (2016)

[24] S. Han, Y. Feng, F. Zhang, C. Yang, Z. Yao, W. Zhao, F. Qiu, L. Yang, Y. Yao, X. Zhuang, X. Feng, Adv. Funct. Mater. 25, 3899 (2015)

[25] Z. Pu, S. Wei, Z. Chen, S. Mu, Appl. Catal. B: Environ 196, 193 (2016)

[26] J.M. McEnaney, J.C. Crompton, J.F. Callejas, E.J. Popczun, A.J. Biacchi, N.S. Lewis, R.E. Schaak, Chem. Mater. 26, 4826 (2014)

[27] H. Zhang, Y. Feng, Y. Zhang, L. Fang, W. Li, Q. Liu, K. Wu, Y. Wang, Chemsuschem 7, 2000 (2014)

[28] Y. Zhang, L. Fan, P. Wang, Y. Yin, X. Zhang, N. Zhang, K. Sun, Nanoscale 9, 17694 (2017)

[29] R.Y. Qiu, R.X. Fei, T.Q. Zhang, X.L. Liu, J. Jin, H.S. Fan, R. Wang, B.B. He, Y.S. Gong, H.W. Wang, Electrochim. Acta 356, $136832(2020)$

[30] M. Yang, Q. Ning, C. Fan, X. Wu, Chin. Chem. Lett. (2020). https ://doi.org/10.1016/j.cclet.2020.07.014

[31] Y. An, S. Chen, M. Zou, L. Geng, X. Sun, X. Zhang, K. Wang, Y. Ma, Rare Met. 38, 1113 (2019)

[32] Z. Gu, J. Guo, Z. Sun, X. Zhao, W. Li, X. Yang, H. Liang, C. Zhao, X. Wu, Sci. Bull. 65, 702 (2020)

[33] Z. Wu, W. Lei, J. Wang, R. Liu, K. Xia, C. Xuan, D. Wang, ACS Appl. Mater. Interfaces 9, 12366 (2017)

[34] G. Park, S. Sim, J. Lee, S.M. Lee, J. Alloys Compd. 639, 296 (2015)

[35] C.T. Buru, O.K. Farha, ACS Appl. Mater. Interfaces 12, 5345 (2020)
[36] S. Wang, J. Hwang, E. Kim, J. Mater. Chem. C 7, 7828 (2019)

[37] M. Nyman, F. Bonhomme, T.M. Alam, M.A. Rodriguez, B.R. Cherry, J.L. Krumhansl, T.M. Nenoff, A.M. Sattler, Science 297, $996(2002)$

[38] X. Yang, X. Feng, H. Tan, H. Zang, X. Wang, Y. Wang, E. Wang, Y. Li, J. Mater. Chem. A 4, 3947 (2016)

[39] H. Lv, Y.V. Geletii, C. Zhao, J.W. Vickers, G. Zhu, Z. Luo, J. Song, T. Lian, D.G. Musaev, C.L. Hill, Chem. Soc. Rev. 41, 7572 (2012)

[40] J. Chen, M. Symes, S. Fan, M. Zheng, H. Miras, Q. Dong, L. Cronin, Adv. Mater. 27, 4649 (2015)

[41] W.H. Casey, B.L. Phillips, Geochim. Cosmochim. Ac. 65, 705 (2001)

[42] L. Zhang, J. Wu, Y. Wang, Y. Long, N. Zhao, J. Xu, J. Am. Chem. Soc. 134, 9879 (2012)

[43] H. Li, Y. Jia, A. Wang, W. Cui, H. Ma, X. Feng, J. Li, Chemistry 20, 499 (2014)

[44] X. Yan, P. Zhu, J. Fei, J. Li, Adv. Mater. 22, 1283 (2010)

[45] Y, Jiang, Y, Shen, J. Dong, S. Tan, Q. Wei, F. Xiong, Q. Li, X. Liao, Z. Liu, Q. An, L. Mai, Adv. Energy Mater. 27, 1900967 (2019)

[46] P. Xiao, M.A. Sk, L. Thia, X. Ge, R.J. Lim, J.Y. Wang, K.H. Lim, X. Wang, Energy Environ. Sci. 7, 2624 (2014)

[47] T. Wu, M. Pi, D. Zhang, S. Chen, J. Power Sources 328, 551 (2016)

[48] J. Senthilnathan, Y.F. Liu, K.S. Rao, M. Yoshimura, Sci. Rep. 4, 4395 (2014)

[49] J. Chen, Z. Mao, L. Zhang, D. Wang, R. Xu, L. Bie, B.D. Fahlman, ACS Nano 11, 12650 (2017)

[50] Y. Zhu, G. Chen, X. Xu, G. Yang, M. Liu, Z. Shao, ACS Catal. 7, 3540 (2017)

[51] X. Wang, P. Sun, J. Qin, J. Wang, Y. Xiao, M. Cao, Nanoscale 8, 10330 (2016)

[52] Z.L. Cai, Z.L, Peng, M.Q. Wang, J.Y. Wu, H.S. Fan, Y.F. Zhang, Rare Met. (2020). https://doi.org/10.1007/s12598-020-01630-y

[53] B. Hou, Y. Wang, D. Liu, Z. Gu, X. Feng, H. Fan, T. Zhang, C. Lü, X. Wu, Adv. Funct. Mater. 28, 1805444 (2018) 